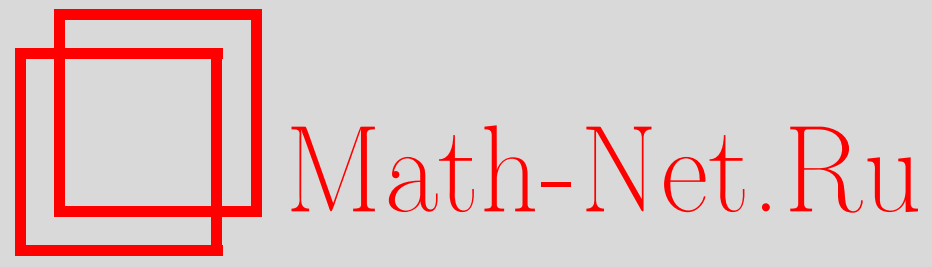

С. Л. Зиглин, Об интегралах в инволюции групп линейных симплектических преобразований и натуральных механических систем с однородным потенциалом, Функи. анализ и его прил., 2000, том 34, выпуск 3, 26-36

DOI: https://doi.org/10.4213/faa309

Использование Общероссийского математического портала MathNet.Ru подразумевает, что вы прочитали и согласны с пользовательским соглашением

http://www. mathnet.ru/rus/agreement

Параметры загрузки:

IP : 35.174 .16 .151

26 апреля 2023 г., 18:28:38

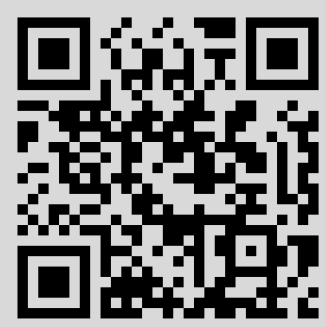


Функциональньй анализ и его приложения

2000, т. 34, вып. 3, с. 26-36

УДК 517.913

\title{
Об интегралах в инволюции групп линейных симплектических преобразований и натуральных механических систем с однородным потенциалом*
}

\author{
(c) 2000. С. Л. Зиглин
}

\section{Введение}

В работе продолжается исследование связи ветвления решений комплексных гамильтоновых систем с отсутствием у них дополнительных (т. е. образующих с гамильтонианом функционально независимую систему) первых интегралов [1]. Основное внимание уделяется многомерным системам (с $n>2$ степенями свободы).

Рассмотрим комплексную гамильтонову систему с $n$ степенями свободы с аналитическим гамильтонианом $H$. Пусть $\Gamma$ - ее комплексная фазовая кривая, не являющаяся положением равновесия. Будем называть приведенной системой в вариациях (для исходной системы) вдоль фазовой кривой Г ограничение системы в нормальных вариациях (той же системы) вдоль этой фазовой кривой на нулевую поверхность уровня ее первого интеграла $d H$ (ср. [2]; см. также [1]).

Будем называть группой монодромии линейной системы в голоморфном векторном расслоении над римановой поверхностью в некоторой ее точке образ естественного антипредставления фундаментальной группы этой поверхности в этой точке в группу линейных преобразований слоя над этой точкой.

Нетрудно видеть [2], что группа монодромии $G_{0}$ приведенной системы в вариациях вдоль фазовой кривой $\Gamma$ является подгруппой группы линейных симплектических преобразований $(2 n-2)$-мерного комплексного линейного симплектического пространства.

Будем называть преобразование $g \in G_{0}$ с собственными значениями $\lambda_{1}, \lambda_{1}^{-1}$, $\ldots, \lambda_{n-1}, \lambda_{n-1}^{-1}$ резонансным [2], если существуют целые числа $k_{1}, \ldots, k_{n-1}$, $\sum k_{i}^{2} \neq 0$, такие, что $\lambda_{1}^{k_{1}} \cdots \lambda_{n-1}^{k_{n-1}}=1$.

Согласно теореме 2 из [2], если группа $G_{0}$ содержит нерезонансное преобразование и другое преобразование, не сохраняющее объединение собственных направлений первого, то исходная гамильтонова система не имеет $n$ функционально независимых мероморфных первых интегралов. Этот факт позволяет доказать неинтегрируемость ряда комплексных гамильтоновых систем с двумя степенями свободы (см., например, [3]).

Как указано в [4], при $n>2$ условие нерезонансности в некоторых случаях может оказаться трудным для проверки.

В настоящей работе указывается класс многомерных гамильтоновых систем, для которых можно получить необходимые условия полной интегрируемости,

*Работа выполнена по программе «Ведущие научные школы», грант РФФИ № 00-1596146. 
т. е. существования $n$ функционально независимых мероморфных первых интегралов в инволюции, в терминах группы монодромии системы в вариациях (или приведенной системы в вариациях), не содержащие многомерных условий нерезонансности. В качестве примера рассматриваются натуральные механические системы с однородным потенциалом $[4,5]$, в частности, задача $n$ тел.

\section{$\S 1$. Общая теория}

Пусть $\left(M^{2 n}, \omega^{2}\right)$ - связное комплексное симплектическое многообразие и $H$ - аналитическая функция на $M$. Рассмотрим гамильтонову систему

$$
\dot{x}=I d H(x), \quad x \in M .
$$

Пусть $Г$ - комплексная фазовая кривая системы (1.1), не являющаяся положением равновесия, $x_{0} \in \Gamma$ и $G$ - группа монодромии системы в вариациях для (1.1) вдоль кривой $\Gamma$ в точке $x_{0}$, т. е. образ естественного антипредставления фундаментальной группы $\pi_{1}\left(\Gamma, x_{0}\right)$ кривой $\Gamma$ в точке $x_{0}$ в группу линейных симплектических преобразований касательного пространства $T_{x_{0}} M$. Предположим, что существуют двумерные линейные взаимно косоортогональные подпространства $L_{1}^{2}, \ldots, L_{n}^{2}$ пространства $T_{x_{0}} M$, инвариантные относительно $G$. Положим $\left\{\left.g\right|_{L_{i}}: g \in G\right\}=G_{i}(i=1, \ldots, n)$.

ТеоремА. Если система (1.1) имеет $n$ функиионально независимьх мероморфных первых интегралов в инволюиии, то при любом $i$ группа $G_{i}$ имеет (непостоянньий) рациональньй первьй интеграл (т. е. рациональную инвариантную функиию).

ДокАЗАтЕЛьство. Предположим, что указанные первые интегралы существуют. Тогда, согласно лемме п. 1.2 из [2], существует $n$ полиномов от $n$ переменных с комплексными коэффициентами, таких, что при подстановке в них указанных первых интегралов младшие однородные части в точке $x_{0}$ получающихся мероморфных функций функционально независимы. (Младшей однородной (квазиоднородной) частью мероморфной функции в точке здесь называется отношение младших однородных (квазиоднородных) частей в этой точке числителя и знаменателя этой функции в ее представлении в виде отношения аналитических функций. Если точка не указывается, то подразумевается, что это начало координат). Очевидно, что эти младшие однородные части являются первыми интегралами группы $G$ и находятся в инволюции. Таким образом, остается доказать следующую лемму.

Пусть $\left(L^{2 n}, \omega^{2}\right)$ есть $2 n$-мерное комплексное линейное симплектическое пространство, $G$ - подгруппа группы его линейных симплектических преобразований и $L_{1}^{2}, \ldots, L_{n}^{2}$ - двумерные линейные взаимно косоортогональные подпространства пространства $L$, инвариантные относительно $G$. Положим, как и ранее, $\left\{\left.g\right|_{L_{i}}: g \in G\right\}=G_{i}(i=1, \ldots, n)$.

Лемма. Если группа $G$ имеет $n$ функционально независимьх рациональных первых интегралов в инволючии, то при любом $i$ группа $G_{i}$ имеет рациональнылй первый интеграл.

ДокАЗАтЕЛЬство. Пусть $\left(e_{i}, f_{i}\right)$ - симплектический базис подпространства $L_{i}$, а $x_{1}, \ldots, x_{n}, y_{1}, \ldots, y_{n}$ - соответствующая симплектическая система координат на $L$. Положим $\left(x_{1}, y_{1}\right)=z_{1},\left(x_{2}, \ldots, x_{n}, y_{2}, \ldots, y_{n}\right)=z_{2}$. 
Предположим, что указанные первые интегралы существуют. Тогда, согласно лемме п. 1.2 из [2], существует $n$ полиномов от $n$ переменных с комплексными коэффициентами, таких, что при подстановке в них этих первых интегралов младшие однородные части $F_{1}, \ldots, F_{n}$ по переменным $z_{2}$ получающихся рациональных функций функционально независимы. Очевидно, они также являются первыми интегралами группы $G$ и находятся в инволюции по переменным $z_{2}$.

Пусть $L^{\prime} \subset L$ - область регулярности функций $F_{1}, \ldots, F_{n}$. Так как размерность нулевой плоскости в $(2 n-2)$-мерном линейном симплектическом пространстве не превосходит $n-1[6]$, то $\operatorname{rank} \partial\left(F_{1}, \ldots, F_{n}\right) / \partial z_{2} \leqslant n-1$ для любой точки $z \in L^{\prime}$.

Пусть $\max _{z \in L^{\prime}} \operatorname{rank} \partial\left(F_{1}, \ldots, F_{n}\right) / \partial z_{2}(z)=r$. (Очевидно, что $\left.r \geqslant n-2.\right)$ Пусть для определенности существует точка $z \in L^{\prime}$, такая, что

$$
\operatorname{rank} \partial\left(F_{1}, \ldots, F_{r}\right) / \partial z_{2}(z)=r .
$$

Тогда для любого $i>r$ функции $f=F_{i}, F=\left(F_{1}, \ldots, F_{r}\right), z_{1}$ функционально зависимы; следовательно [7, гл. Х, предложение 10], они алгебраически зависимы, т.е. существует полином $P\left(X_{1}, \ldots, X_{r+3}\right)$ с ненулевыми комплексными коэффициентами, такой, что $P\left(f, F, z_{1}\right)=0$. Очевидно, что $\partial P / \partial X_{1} \neq 0$ (иначе функции $F, z_{1}$ были бы функционально зависимы, вопреки равенству (1.2)). Выберем полином $P$ так, чтобы его степень по переменной $X_{1}$ была минимальной. Тогда $\partial P / \partial X_{1}\left(f, F, z_{1}\right) \neq 0$.

Выберем точку $z \in L^{\prime}$ так, что

1) $d F_{1}(z), \ldots, d F_{n}(z)$ линейно независимы,

2) $\operatorname{rank} \partial F / \partial z_{2}(z)=r$

3) $\partial P / \partial X_{1}\left(f(z), F(z), z_{1}(z)\right) \neq 0$.

Тогда на множестве $N=\left\{z^{\prime} \in L^{\prime}: F\left(z^{\prime}\right)=F(z)\right\}$ функция $f$ удовлетворяет уравнению

$$
Q\left(f, z_{1}\right)=\sum_{j=0}^{k} a_{j}\left(z_{1}\right) f^{j}=0
$$

где $a_{j}$ - полиномы, $k>0, a_{k}\left(z_{1}\right) \not \equiv 0$ на $N$. (Далее ср. [8], доказательство теоремы Лиувилля [9] об отсутствии дополнительного алгебраического первого интеграла в задаче о движении динамически симметричного тяжелого твердого тела около неподвижной точки в случае отсутствия полиномиального квазиоднородного.)

Выберем полином $Q$ так, чтобы его степень $k$ по переменной $f$ была минимальной. Из инвариантности множества $N$ и функции $f$ относительно группы $G$ следует, что для любого $g \in G$ функция $f$ удовлетворяет на множестве $N$ также уравнению

$$
\sum_{j=0}^{k} a_{j}\left(z_{1} \circ g\right) f^{j}=0 .
$$

Умножая уравнение (1.3) на $a_{k}\left(z_{1} \circ g\right)$, а уравнение $(1.4)-$ на $a_{k}\left(z_{1}\right)$ и вычитая их одно из другого, получаем, что функция $f$ удовлетворяет на множестве $N$ уравнению

$$
\sum_{j=0}^{k-1} b_{j}\left(z_{1}\right) f^{j}=0, \quad b_{j}\left(z_{1}\right)=a_{j}\left(z_{1}\right) a_{k}\left(z_{1} \circ g\right)-a_{j}\left(z_{1} \circ g\right) a_{k}\left(z_{1}\right) .
$$


Из инвариантности подпространств $L_{1}, \ldots, L_{n}$ относительно $G$ следует, что функции $z_{1} \circ g$ являются линейными комбинациями функций $z_{1}$. Из минимальности числа $k$ получаем, что $b_{j}\left(z_{1}\right) \equiv 0$ на $N$, а значит, и на множестве $N_{1}=\pi(N)$, где $\pi: L \rightarrow L_{1}$ - естественная проекция.

Из условия 2) следует, что множество $N_{1}$ содержит открытое подмножество; следовательно, $b_{j}=0$, т. е. $a_{j}\left(z_{1}\right) / a_{k}\left(z_{1}\right)=a_{j}\left(z_{1} \circ g_{1}\right) / a_{k}\left(z_{1} \circ g_{1}\right)$ на $L_{1}$, где $g_{1}=\left.g\right|_{L_{1}}$, т. е. рациональные функции $a_{j}\left(z_{1}\right) / a_{k}\left(z_{1}\right)(j=1, \ldots, k-1)$ являются первыми интегралами группы $G_{1}$.

Покажем, что не все они являются постоянными. Действительно, в противном случае функция $f$ принимала бы на множестве $N^{\prime}=\left\{z^{\prime} \in N: a_{k}\left(z_{1}\left(z^{\prime}\right)\right) \neq 0\right\}$ конечное число значений. Но из условий 1$), 2$ ) следует, что сколь угодно близко к точке $z$ существует точка $z^{\prime} \in N^{\prime}$, в которой дифференциалы $d F_{1}\left(z^{\prime}\right), \ldots, d F_{n}\left(z^{\prime}\right)$ линейно независимы. Для любой окрестности $U \subset L^{\prime}$ точки $z^{\prime}$ функция $f$ принимает на множестве $U \cap N^{\prime}$ бесконечное число значений. Лемма доказана.

ЗАмЕчАниЕ. Аналогичная теорема справедлива и для группы монодромии $G_{0}$ приведенной системы в вариациях вдоль $Г$, т.е. для группы монодромии ограничения системы в нормальных вариациях вдоль Г на нулевую поверхность уровня ее первого интеграла $d H$ (ср. [2]). Действительно, согласно предложению п. 1.2 из [2], если система (1.1) имеет $r$ дополнительных (т. е. функционально независимых с $H$ ) мероморфных первых интегралов, то группа $G_{0}$ имеет $r$ функционально независимых рациональных первых интегралов. Из способа доказательства этого предложения, приведенного в [2], следует, что если исходные первые интегралы находятся в инволюции, то полученные первые интегралы также будут в инволюции. Если $r=n-1$ и группа $G_{0}$ имеет $n-1$ двумерных взаимно косоортогональных инвариантных подпространств, то применима доказанная выше лемма.

\section{§2. Натуральные механические системы с однородным потенциалом}

Пусть $D$ - область в $n$-мерном комплексном координатном пространстве $\mathbb{C}^{n}$, инвариантная относительно умножения на ненулевые комплексные числа и такая, что $\operatorname{Re} D=D \cap \mathbb{R}^{n} \neq \varnothing$, и $V$ - вещественно-аналитическая однородная функция в $D$ целой степени $k$.

Будем использовать следующие обозначения. Если $x=\left(x_{1}, \ldots, x_{m}\right) \in \mathbb{C}^{m}$, $y=\left(y_{1}, \ldots, y_{m}\right) \in \mathbb{C}^{m}$, то $\langle x, y\rangle=\sum x_{i} y_{i}, x^{2}=\langle x, x\rangle$.

Рассмотрим, следуя $[4,5]$, гамильтонову систему

$$
\dot{q}=\frac{\partial H}{\partial p}, \quad \dot{p}=-\frac{\partial H}{\partial q}, \quad q \in D, p \in \mathbb{C}^{n}
$$

где $H=p^{2} / 2+V(q)$.

Пусть $c \in \operatorname{Re} D$ - ненулевое решение системы уравнений

$$
c=\frac{\partial V}{\partial q}(c) .
$$

ЗАмЕчАниЕ. При $k=0$ таких решений нет, так как при вещественных $q$ имеем $q \perp \partial V / \partial q$.

Рассмотрим прямолинейное решение

$$
q=c \phi(t), \quad p=c \dot{\phi}(t)
$$


системы (2.1), где $\phi-$ решение дифференциального уравнения $\ddot{\phi}+\phi^{k-1}=0$ с начальным условием $\phi(0)=1, \dot{\phi}(0)=0$.

Система в вариациях вдоль комплексифицированной фазовой кривой $\Gamma$ решения (2.3) имеет вид

$$
\dot{\xi}=\eta, \quad \dot{\eta}=-\phi^{k-2}(t) \frac{\partial^{2} V}{\partial q^{2}}(c) \xi, \quad \xi, \eta \in \mathbb{C}^{n} .
$$

В ортонормированном собственном базисе матрицы $\partial^{2} V / \partial q^{2}(c)$ система $(2.4)$ принимает вид

$$
\dot{\xi}_{i}=\eta_{i}, \quad \dot{\eta}_{i}=-\lambda_{i} \phi^{k-2}(t) \xi_{i} \quad(i=1, \ldots, n),
$$

где $\lambda_{1}, \ldots, \lambda_{n}$ - собственные значения матрицы $\partial^{2} V / \partial q^{2}(c)$, или

$$
\ddot{\xi}_{i}+\lambda_{i} \phi^{k-2}(t) \xi_{i}=0 \quad(i=1, \ldots, n) .
$$

Из теоремы $\S 1$ вытекает

Теорема. Если система (2.1) имеет $n$ функиионально независимьх мероморфных первых интегралов в инволючии, то группа монодромии каждого из уравнений системы (2.5) имеет рачиональный первый интеграл.

Пусть $k \neq 0, \pm 2$.

ЗАмечАниЕ. При $k= \pm 2$ каждое из уравнений системы (2.5) имеет линейный или квадратичный первый интеграл. (При $k=2$ это очевидно, а при $k=-2$ следует из цикличности группы монодромии, которая, в свою очередь, следует из тождественности преобразований монодромии, соответствующих обходам бесконечно удаленных по координатам точек на фазовой кривой. Последняя проверяется непосредственными выкладками).

В $[4,5]$ показано, что если $i$-е уравнение системы $(2.5)$ имеет рациональный первый интеграл, то числа $\mu_{i}, \nu_{i}$, определяемые из квадратных уравнений

$$
\mu_{i}^{2}-2\left[\cos (2 \pi / k)+2 \cos ^{2}\left(\pi \omega_{i} / 2 k\right)\right] \mu_{i}+1=0, \quad \nu_{i}^{2}-2 \nu_{i} \cos 2 \pi \omega_{i}+1=0,
$$

где $\omega_{i}=\sqrt{(k-2)^{2}+8 k \lambda_{i}}$, являются корнями из единицы. (В действительности в $[4,5]$ рассматривались полиномиальные первые интегралы, однако результаты с помощью леммы п. 1.3 из [2] переносятся и на случай рациональных первых интегралов).

ЗАмЕчАниЕ $[4,5]$. Из этого утверждения следует, что числа $\omega_{i}$ рациональны и удовлетворяют неравенству

$$
\cos (2 \pi / k)+2 \cos ^{2}\left(\pi \omega_{i} / 2 k\right) \leqslant 1 .
$$

\section{Отсюда вытекает}

ПреДЛОЖЕНИЕ 1. Если система (2.1) имеет $n$ функиионально независимьх мероморФньх первых интегралов в инволючии, то все числа $\mu_{i}, \nu_{i}(i=$ $1, \ldots, n)$ являются корнями из единицьл.

ЗАмЕчАниЕ. В $[4,5]$ показано, что одно из чисел $\lambda_{i}$, для определенности $\lambda_{n}$, равно $k-1$, и для существования у системы (2.1) дополнительного аналитического первого интеграла необходимо, чтобы наборы чисел $\mu_{1}, \ldots, \mu_{n-1}$ и $\nu_{1}, \ldots, \nu_{n-1}$ были резонансными, т. е. чтобы существовали целые числа $k_{1}, \ldots$, $k_{n-1}, l_{1}, \ldots, l_{n-1}, \sum k_{i}^{2} \neq 0, \sum l_{i}^{2} \neq 0$, такие, что $\mu_{1}^{k_{1}} \cdots \mu_{n-1}^{k_{n-1}}=1, \nu_{1}^{l_{1}} \cdots \nu_{n-1}^{l_{l_{n-1}}}=1$ 
(последнее равенство эквивалентно рациональной зависимости чисел $\omega_{1}, \ldots, \omega_{n}$ $[5])$.

ПримеР [5]. Рассмотрим систему $n$ точек на прямой с потенциалом

$$
V(q)=\left(-q_{1}\right)^{k}+\left(q_{1}-q_{2}\right)^{k}+\cdots+\left(q_{n-1}-q_{n}\right)^{k}+q_{n}^{k},
$$

где $k>2$ - четное, а $n \geqslant 1$ - нечетное натуральные числа.

В этом случае уравнение (2.2) имеет решение

$$
c_{1}=-c_{3}=c_{5}=\cdots=(1 / 2 k)^{1 /(k-2)}, \quad c_{2}=c_{4}=\cdots=0 .
$$

Как показано в [5], собственные значения матрицы $\partial^{2} V / \partial q^{2}(c)$ имеют вид

$$
\lambda_{j}=2(k-1) \sin ^{2}\left(\frac{j \pi}{2(n+1)}\right) \quad(j=1, \ldots, n) .
$$

В [5] было показано, что при $k=4$ и $n=1,3,5$ числа $\omega_{1}, \ldots, \omega_{n}$ рационально независимы и, согласно предыдущему замечанию, система (2.1) не имеет дополнительного аналитического первого интеграла, и высказано предположение, что это утверждение справедливо при всех четных $k$ и нечетных $n$.

ПрЕДЛОЖЕНИЕ 2. При четных $k>2$ и нечетных $n \geqslant 3$ система (2.1) с потенииалом (2.7) не имеет $n$ функиионально независимьх мероморфньх первых интегралов в инволюиии ни в какой области, содержащей начало координат.

ДокАЗАтЕльство. Покажем, что если такие первые интегралы существуют в какой-либо области, содержащей начало координат, то они существуют во всем комплексном фазовом пространстве $\mathbb{C}^{2 n}$ (ср. [3, доказательство теоремы из $§ 4])$. Действительно, предположим, что такие первые интегралы существуют. Тогда, согласно лемме п. 1.2 из [2], существует $n$ полиномов от $n$ переменных с комплексными коэффициентами, таких, что при подстановке в них этих первых интегралов младшие квазиоднородные части получающихся мероморфных функций по переменным $p, q$, где переменным $p$ приписывается вес $k$, а $q$ - вес 2 , функционально независимы. Очевидно, они также находятся в инволюции и, как следует из квазиоднородности гамильтониана $H$, являются первыми интегралами системы (2.1), что и требовалось доказать. Таким образом, остается показать, что указанных первых интегралов не существует во всем комплексном фазовом пространстве $\mathbb{C}^{2 n}$.

Как показано в [4], при $k>2$ и

$$
1<\lambda_{i}<k-1
$$

неравенство (2.6) невозможно.

Полагая $n=2 l-1$, получаем

$$
\lambda_{l-1}=2(k-1) \sin ^{2}\left(\frac{\pi}{4}-\frac{\pi}{4 l}\right)=(k-1)\left(1-\sin \frac{\pi}{2 l}\right) .
$$

Так как $3(1-\sin (\pi / 6))>1$, то при $l \geqslant 3, i=l-1$ число $\lambda_{i}$ удовлетворяет неравенству (2.8). Это доказывает предложение 2 при $n \geqslant 5$.

Поскольку $\sin ^{2}(\pi / 8)=(1-1 / \sqrt{2}) / 2$ иррационально, при $n=3$ число $\omega_{1}$ иррационально, что доказывает предложение 2 при $n=3$. 


\section{§3. Задача $n$ тел}

В [4] доказано отсутствие аналитического первого интеграла в задаче трех тел на комплексной прямой, образующего с интегралами энергии и импульса функционально независимую систему и находящегося с ними в инволюции. В настоящем параграфе доказывается отсутствие полного набора мероморфных первых интегралов в инволюции в задаче трех тел в комплексном пространстве произвольного числа измерений и в задаче $n>3$ тел в комплексном пространстве более одного измерения.

3.1. Задача трех тел. Пусть $D$ - область в $3 k$-мерном комплексном координатном пространстве $\mathbb{C}^{3 k}(k \geqslant 1)$ с координатами $q=\left(q_{1}, q_{2}, q_{3}\right), q_{i}=$ $\left(q_{i}^{1}, \ldots, q_{i}^{k}\right)(i=1,2,3)$, задаваемая неравенствами

$$
\left|\left(q_{i}^{2}-q_{j}^{2}\right)^{2}+\cdots+\left(q_{i}^{k}-q_{j}^{k}\right)^{2}\right|<\left|q_{i}^{1}-q_{j}^{1}\right|^{2} \quad(i<j \leqslant 3) .
$$

Положим

$$
L^{3}=\left\{q \in U: q_{i}^{2}=\cdots=q_{i}^{k}=0, i=1,2,3\right\} .
$$

В области $D$ функции $1 / r_{i, j}(i<j \leqslant 3)$, определяемые равенствами

$$
r_{i, j}^{2}=\left(q_{i}-q_{j}\right)^{2},\left.\quad r_{i, j}\right|_{L}=q_{i}^{1}-q_{j}^{1},
$$

однозначны и аналитичны.

Рассмотрим гамильтонову систему

$$
\dot{q}=\frac{\partial H}{\partial p}, \quad \dot{p}=-\frac{\partial H}{\partial q}, \quad q \in D, p \in \mathbb{C}^{3 n},
$$

где

$$
\begin{gathered}
p=\left(p_{1}, p_{2}, p_{3}\right), \quad p_{i}=\left(p_{i}^{1}, \ldots, p_{i}^{k}\right) \quad(i=1,2,3), \\
H=\sum_{i} \frac{p_{i}^{2}}{2 m_{i}}+W, \quad W=g \sum_{i<j} \frac{m_{i} m_{j}}{r_{i, j}}, \quad m_{i}>0 \quad(i=1,2,3), \quad g<0 .
\end{gathered}
$$

В связной компоненте вещественной части фазового пространства, определяемой неравенствами $q_{1}>q_{2}>q_{3}$, система (3.1) описывает движение трех точек с массами $m_{i}$, притягивающихся по закону Ньютона.

Tеорема (ср. [10-13]). В положительном октанте трехмерного координатного пространства $\mathbb{R}^{3}$ с координатами $m=\left(m_{1}, m_{2}, m_{3}\right)$ существует окрестность $U$ объединения $S$ биссекторных плоскостей $m_{i}=m_{j}(i \neq j) c$ вькинутылми прямьми $m_{k} / m_{i}=11 / 12,1 / 4,1 / 24(i \neq k \neq j)$, такая, что при $m \in U$ система (3.1) не имеет $3 n$ функиионально независимых мероморфных первых интегралов в инволюиии.

ДокАЗАТЕЛЬСтво. Сделаем каноническую замену переменных

$$
p_{i}=\sqrt{m_{i}} P_{i}=\sqrt{m_{i}}\left(P_{i}^{1}, \ldots, P_{i}^{k}\right), \quad q_{i}=\frac{Q_{i}}{\sqrt{m_{i}}}=\frac{\left(Q_{i}^{1}, \ldots, Q_{i}^{k}\right)}{\sqrt{m_{i}}} \quad(i=1,2,3) .
$$

Тогда система (3.1) примет вид

$$
\dot{Q}=\frac{\partial F}{\partial P}, \quad \dot{P}=-\frac{\partial F}{\partial Q},
$$


где

$$
F=\frac{1}{2} \sum_{i} P_{i}^{2}+V, \quad V\left(Q_{1}, Q_{2}, Q_{3}\right)=W\left(\frac{Q_{1}}{\sqrt{m_{1}}}, \frac{Q_{2}}{\sqrt{m_{2}}}, \frac{Q_{3}}{\sqrt{m_{3}}}\right) .
$$

Рассмотрим решение системы уравнений $c=\partial V / \partial Q(c)$ вида $c=\left(c_{1}, c_{2}, c_{3}\right)$, $c_{i}=\left(b_{i}, 0, \ldots, 0\right), i=(1,2,3)$, где числа $b_{1}, b_{2}, b_{3}$ удовлетворяют системе уравнений

$$
b_{i}=\partial V / \partial Q_{i}^{1}(c) \quad(i=1,2,3) .
$$

Пусть $m \in S$ и для определенности $m_{1}=m_{3}$. Выберем единицы измерения так, что $m_{1}=1$. Тогда система (3.3) имеет решение [4]

$$
b_{2}=0, \quad b_{1}=-b_{3}=-\left[g\left(m_{2}+1 / 4\right)\right]^{1 / 3} .
$$

Покажем, что собственные значения матрицы

$$
B=\partial^{2} V / \partial\left(Q^{1}\right)^{2}(c)
$$

не равны единице и, следовательно, при близких значениях $m$ система (3.3) имеет решение, близкое к решению (3.4). Действительно, так как ограничение функции $V$ на многообразие $Q_{i}^{2}=\cdots=Q_{i}^{n}=0(i=1,2,3)$ зависит только от двух линейных функций $q_{1}^{1}-q_{1}^{2}$ и $q_{2}^{1}-q_{3}^{1}$, то одно из собственных значений матрицы $B$ равно нулю. Другое, согласно замечанию в конце $\S 2$, равно -2 . Следовательно, третье собственное значение равно

$$
\lambda=\operatorname{trace} A+2=-8\left(m_{2}+2\right) /\left(4 m_{2}+1\right) \neq 1,
$$

что и требовалось доказать.

Согласно предложению $1 \S 2$, для существования у системы (3.2) полного набора мероморфных первых интегралов в инволюции необходимо, чтобы собственные значения матрицы $A=\partial^{2} V / \partial Q^{2}(c)$ удовлетворяли неравенству (2.6). Последнее при $k=-1$ эквивалентно равенству $\lambda_{i}=1-l(l+1) / 2$, где $l$ — целое число [4].

Так как спектр матрицы $B$ содержится в спектре матрицы $A$, то для доказательства теоремы остается проверить, что $\lambda \neq 1-l(l+1) / 2$, где $l$ целое, а это непосредственно следует из условия.

3.2. Задача $\boldsymbol{n}$ тел. Введем следующие обозначения. Если $x=\left(x_{1}, \ldots, x_{m}\right) \in$ $\mathbb{C}^{m}$, то $\|x\|=\sqrt{\sum\left|x_{i}\right|^{2}}$.

Пусть $D$ - область $k n$-мерного комплексного координатного пространства $\mathbb{C}^{k n}(k>1, n>2)$ с координатами $q=\left(q_{1}, \ldots, q_{n}\right), q_{i}=\left(q_{i}^{1}, \ldots, q_{i}^{k}\right)(i=$ $1, \ldots, n)$, задаваемая неравенством

$$
\left\|q-q_{1}^{1} a\right\|<\frac{2}{5}\left|q_{1}^{1}\right| \sin ^{2} \frac{\pi}{n-1},
$$

где

$$
\begin{gathered}
a=\left(a_{1}, \ldots, a_{n}\right), \quad a_{i}=\left(a_{i}^{1}, \ldots, a_{i}^{k}\right) \quad(i=1, \ldots, n), \\
a_{i}^{3}=\cdots=a_{i}^{k}=0 \quad(i=1, \ldots, n), \\
a_{i}^{1}=\cos \frac{2 \pi i}{n-1}, \quad a_{i}^{2}=\sin \frac{2 \pi i}{n-1} \quad(i=1, \ldots, n-1), \quad a_{n}=0 .
\end{gathered}
$$


При $i \neq j$ имеем

$$
\begin{aligned}
\left|\left(q_{i}-q_{j}\right)^{2}-\left(q_{1}^{1}\right)^{2}\left(a_{i}-a_{j}\right)^{2}\right| & =\left|\left\langle q_{i}-q_{j}-q_{1}^{1}\left(a_{i}-a_{j}\right), q_{i}-q_{j}+q_{1}^{1}\left(a_{i}-a_{j}\right)\right\rangle\right| \\
& \leqslant\left\|q_{i}-q_{j}-q_{1}^{1}\left(a_{i}-a_{j}\right)\right\|\left\|q_{i}-q_{j}+q_{1}^{1}\left(a_{i}-a_{j}\right)\right\| \\
& <\frac{4}{5}\left|q_{1}^{1}\right|^{2} \sin ^{2} \frac{\pi}{n-1}\left(\frac{4}{5} \sin ^{2} \frac{\pi}{n-1}+4\right) \\
& <4\left|q_{1}^{1}\right|^{2} \sin ^{2} \frac{\pi}{n-1} \leqslant\left|q_{1}^{1}\right|^{2}\left\|a_{i}-a_{j}\right\|^{2} ;
\end{aligned}
$$

следовательно, функции $1 / r_{i, j}(i<j)$, определяемые равенствами

$$
r_{i, j}^{2}=\left(q_{i}-q_{j}\right)^{2}, \quad r_{i, j}(a)=\left\|a_{i}-a_{j}\right\|,
$$

в области $D$ однозначны и аналитичны.

Рассмотрим гамильтонову систему

$$
\dot{q}=\frac{\partial H}{\partial p}, \quad \dot{p}=-\frac{\partial H}{\partial q}, \quad q \in D, p \in \mathbb{C}^{k n},
$$

где

$$
\begin{gathered}
p=\left(p_{1}, \ldots, p_{n}\right), \quad p_{i}=\left(p_{i}^{1}, \ldots, p_{i}^{k}\right)(i=1, \ldots, n), \\
H=\frac{1}{2} \sum_{i} \frac{p_{i}^{2}}{m_{i}}+W, \quad W=g \sum_{i<j} \frac{m_{i} m_{j}}{r_{i, j}}, \quad m_{i}>0(i=1, \ldots, n), \quad g<0 .
\end{gathered}
$$

В связной компоненте вещественной части фазового пространства, определяемой неравенством $q_{1}^{1}>0$, система (3.5) описывает движение $n$ точек в $k$-мерном координатном пространстве, притягивающихся по закону Ньютона.

Теорема (ср. [14], обобщение Пенлеве на задачу $n$ тел теоремы Брунса об отсутствии дополнительного алгебраического первого интеграла задачи трех тел). При фиксированных $m_{1}=\cdots=m_{n-1}$ существует не более конечного числа значений $m_{n}$, при которых система (3.5) имеет $k n$ функционально независимых мероморфных первых интегралов в инволюиии.

ДокАзАтельство. Сделаем каноническую замену переменных $p_{i}=\sqrt{m_{i}} P_{i}$, $q_{i}=Q_{i} / \sqrt{m_{i}}(i=1, \ldots, n)$. Тогда в новых переменных гамильтониан примет вид

$$
F=\frac{1}{2} \sum_{i} P_{i}^{2}+V, \quad V\left(Q_{1}, \ldots, Q_{n}\right)=W\left(\frac{Q_{1}}{\sqrt{m_{1}}}, \ldots, \frac{Q_{n}}{\sqrt{m_{n}}}\right) .
$$

Выберем единицы измерения так, что $m_{1}=\cdots=m_{n-1}=1$.

Уравнение $c=\partial V / \partial Q(c)$ имеет решение $c=b a$, где

$$
b=-\left[g\left(\frac{1}{4} \sum_{i=1}^{n-2} \sin ^{-1} \frac{\pi i}{n-1}+m_{n}\right)\right]^{1 / 3} .
$$

Из сказанного в конце п. 3.1 следует, что, если

$$
\operatorname{Spec} \frac{\partial^{2} V}{\partial Q^{2}}(c) \not \subset \mathbb{Z},
$$

то система (3.5) не имеет $k n$ функционально независимых мероморфных первых интегралов в инволюции. 
TaK каK

$$
\operatorname{Spec} \frac{\partial^{2} V}{\partial Q^{2}}(c) \supset \operatorname{Spec} \frac{\partial^{2} V}{\partial \bar{Q}^{2}}(c),
$$

где

$$
\bar{Q}=\left(\bar{Q}_{1}, \ldots, \bar{Q}_{n}\right), \quad \bar{Q}_{i}=\left(Q_{i}^{1}, Q_{i}^{2}\right) \quad(i=1, \ldots, n),
$$

то для доказательства теоремы остается показать, что существует не более конечного числа значений $m_{n}$, при которых

$$
p=\operatorname{trace} \frac{\partial^{2} V}{\partial \bar{Q}^{2}}(c) \in \mathbb{Z} .
$$

Поскольку

$$
p=-(n-1) \frac{m_{n}+\frac{1}{8} \sum_{i=1}^{n-2} \sin ^{-3} \frac{\pi i}{n-1}+1}{m_{n}+\frac{1}{4} \sum_{i=1}^{n-2} \sin ^{-1} \frac{\pi i}{n-1}},
$$

остается показать, что

$$
1+\frac{1}{8} \sum_{i=1}^{n-2} \sin ^{-3} \frac{\pi i}{n-1}>\frac{1}{4} \sum_{i=1}^{n-2} \sin ^{-1} \frac{\pi i}{n-1},
$$

T. e.

$$
S=\sum_{i=1}^{n-2} \cos \frac{2 \pi i}{n-1} \sin ^{-3} \frac{\pi i}{n-1}>-8 .
$$

Имеем

$$
\begin{aligned}
S & \geqslant 2 \sum_{i=1}^{[(n-1) / 2]} \cos \frac{2 \pi i}{n-1} \sin ^{-3} \frac{\pi i}{n-1} \geqslant 2 \cos \frac{2 \pi}{n-1}[(n-1) / 2] \sin ^{-3} \frac{\pi}{n-1}[(n-1) / 2] \\
& \geqslant-2 \sin ^{-3}\left(\frac{\pi}{2}-\frac{\pi}{2(n-1)}\right) \geqslant-2 \sin ^{-3} \frac{\pi}{4}=-4 \sqrt{2}>-8
\end{aligned}
$$

что и требовалось доказать.

Пользуясь случаем, исправим замеченную неточность в [15].

На стр. 4 вместо «все, кроме, может быть, счетного числа» должно быть «все, кроме не более чем счетного числа».

Автор благодарен В. И. Арнольду и А. И. Нейштадту за ценные замечания.

ПРИМЕЧАНИЕ ПРИ КОРРЕКТУРЕ. Когда настоящая работа находилась в печати, автору стало известно о работе [16], в которой содержится утверждение (теорема 5.1), более сильное, чем предложение $1 \S 2$ настоящей работы: при $k \neq \pm 1$ числа $\lambda_{i}$ должны удовлетворять более жестким оганичениям, чем указано в формулировке этого предположения. Автор благодарен Ж. Ж. МоралесуРуизу за присланную книгу [16] и любезные разъяснения. 


\section{ЛИТЕРАТУРА}

1. Козлов B. В. Симметрии, топология и резонансы в гамильтоновой механике. Изд-во Удмуртского гос. ун-та, Ижевск, 1995.

2. Зиглин C. Л. Ветвление решений и несуществование первых интегралов в гамильтоновой механике. І. Функц. анализ и его прил., 16, вып. 3, 30-41 (1982).

3. Зиглин C. Л. Ветвление решений и несуществование первых интегралов в гамильтоновой механике. ІІ. Функц. анализ и его прил. 17, вып. 1, 8-24 (1983).

4. Yoshida H. A criterion for the non-existence of an additional integral in Hamiltonian systems with homogeneous potential. Phys. D, 29, 128-142 (1987).

5. Yoshida H. A criterion for the non-existence of an additional analitic integral in Hamiltonian systems with $n$ degrees of freedom. Phys. Lett. A, 141, 108-112 (1989).

6. Арнольд В. И. Математические методы классической механики. Наука, М., 1974.

7. Ленг С. Алгебра. Мир, М., 1968.

8. Полубаринова-Кочина П. Я. Об однозначных решениях и алгебраических интегралах задачи о вращении тяжелого твердого тела около неподвижной точки. В кн.: Движение твердого тела около неподвижной точки. Сборник, посвященный памяти С. В. Ковалевской. Изд-во АН СССР, М.-Л., 1940, с. 157-186.

9. Liouville $R$. Sur la mouvement d'un corps solide pesant suspendu par l'un ses points. Acta Math., 20, 239-284 (1896).

10. Алексеев B. М. Квазислучайные динамические системы, 2. Одномерные нелинейные колебания в периодически возмущаемом поле. Матем. сб., 77, № 4, 545-601 (1968).

11. Алексеев B. М. Квазислучайные динамические системы, 3. Квазислучайные колебания одномерных осцилляторов. Матем. сб., 78, № 1, 3-50 (1969).

12. Алексеев B. М. Финальные движения в задаче трех тел и символическая динамика. УМН, 36, вып. 4, 161-176 (1981).

13. Libre J., Simo C. Oscillatory solutions in the planar restricted three-body problem. Math. Ann., 248, No. 2, 153-184 (1980).

14. Уиттекер E. T. Аналитическая динамика. ОНТИ, М.-Л., 1937.

15. Зиглин C. Л. Об отсутствии дополнительного первого интеграла в некоторых задачах динамики. Функц. анализ и его прил., 31, вып. 1, 3-11 (1997).

16. Morales-Ruiz J. J. Differential Galois theory and nonintegrability of Hamiltonian systems. Progress in Math., vol. 179, Birkhäuser, 1999. 